\title{
Liver cirrhosis and the anticoagulant treatment - from guided indications to daily practice - case report
}

\author{
Nicoleta Dima ${ }^{*}$, , Luminiţa Gina Vâţă ${ }^{2,5}$, Elena Rezuş, ${ }^{3,5}$ Ciprian Rezuş ${ }^{1,5}$ Anca \\ $\operatorname{Trifan}^{4,5}$
}

${ }^{1} \mathrm{III}^{\text {rd }}$ Medical Clinic, "Sf. Spiridon" Clinical Emergency Hospital, lasi, ${ }^{2} \mathrm{II}^{\text {rd }}$ Medical Clinic, "Sf. Spiridon" Clinical Emergency Hospital, lasi, ${ }^{3} 1^{\text {st }}$ Rheumatology Clinic, Clinical Rehabilitation Hospital, lasi, Romania, ${ }^{4}$ Gastroenterology and Hepatology Center, "Sf. Spiridon" Clinical Emergency Hospital, lasi, 5 "Grigore T. Popa" University of Medicine and Pharmacy, lasi, Romania.

\begin{abstract}
This is the case of a 64-year old hypertensive, diabetic patient, known to have liver cirrhosis, with a history of pulmonary thromboembolism, with permanent electrical cardiac stimulation, who was hospitalized for a newly diagnosed giant popliteal hematoma. The patient is currently discharged and following oral anticoagulant treatment.

Keywords: liver cirrhosis, oral anticoagulant treatment, permanent electrical cardiac stimulation, type 2 diabetes mellitus, pulmonary thromboembolism
\end{abstract}

\section{Introduction}

The liver cirrhosis, an irreversible and diffuse illness of the liver is an important cause for morbidity and mortality on a national and international scale, with a high impact on patients, their families, the medical system, society and economy.

During the last decade, a significant increase of its incidence is noticed all over the world, even though not everywhere the same factors are involved. Medical literature highlights that roughly $0.1 \%$ of Europe's population suffers from liver cirrhosis, which corresponds to 14-26 new cases per 100 inhabitants per year, or an estimated number of 170,000 deaths per year [1].

Received: March 2015; Accepted after review: March 2015; Published: March 2015

${ }^{*}$ Corresponding author: Nicoleta Dima, MD, PhD, III ${ }^{\text {rd }}$ Medical Clinic, "Sf. Spiridon" Clinical Emergency Hospital, lasi, Romania. Email: nicoleta2006r@yahoo.com
According to the most recent studies, Romania holds the $2^{\text {nd }}$ place in Europe (after the Republic of Moldavia) regarding the mortality caused by cirrhosis (women being most affected).

The cirrhosis can develop in the form of the compensated stage with minimum clinicalbiological changes or as decompensated cirrhosis, when specific changes for parenchymatous and vascular decompensation are present.

As a consequence of hepatic dysfunction, during cirrhosis development, manifestations may appear at the level of other organs or systems too, most significantly affecting the cardiovascular system, with therapeutic and prognostic implications.

Oral anticoagulants, as acenocumarol, are frequently used in daily practice for the prevention and treatment of thromboembolic diseases. They are characterized by an interindividual variability concerning the treatment response. The possible complications of the oral anticoagulant treatment include severe hemorrhages or, on the contrary, the absence of the efficiency, resulting either from an 
excessive anticoagulation, or from an insufficient level of anticoagulation.

\section{Case report}

A 64 year-old patient was admitted for a giant hematoma in the right popliteal region, the patient being currently discharged and following oral anticoagulant treatment. Some of his personal pathological antecedents are: pulmonary thromboembolism (2003), essential arterial hypertension (diagnosed in 2007, BP $\max =190 / 100 \mathrm{mmHg}$ ), diabetes mellitus type 2 since 2009, in the stage of neuropathic complications (the patient is currently under diet) permanent electrical cardiac stimulation type VVI in 2009 (for total atrioventricular block with syncope), toxic liver cirrhosis since 2009 (a superior digestive endoscopy revealing at that time $2^{\text {nd }}$ degree esophageal varices, chronic kidney disease since 2011 secondary to mixed nephropathy and an episode of bilateral epistaxis in October 2014, given the chronic hepatic illness and the oral anticoagulant treatment.

The objective examination revealed: a relatively good general state, afebrile, conscious patient, a normal weight $(\mathrm{BMI}=$ 23,7 $\mathrm{kg} / \mathrm{m}^{2}$ ), mucous-cutaneous pallor, abdomino-thoracic collateral circulation (Figure 1), giant hematoma in the right popliteal region extending to the level of the inferior half of the calf and the inferior third of the thigh (approximately $21 / 9 \mathrm{~cm}$ ), lipodermatosclerosis of the inferior members, walk with unilateral support, due to the functional impotence of the right inferior limb, emphysematous thorax, $\mathrm{SpO}_{2}=96 \%$ in the ambient air, apexian shock left intercostal space VII on anterior axillary line, $B P=140 / 90 \mathrm{mmHg}, A V \approx 69$ beats/minute, arrhythmic cardiac noises, 4/6 degree mitral systolic murmur with axillary irradiation, 4/6 degree tricuspid systolic murmur, aortic atheromatosis murmur, turgescent jugular veins, supple abdomen, mobile when breathing, sensitive at touch in the right hypochondrium, without signs of peritoneal irritation, liver with sharp inferior margin, $4 \mathrm{~cm}$ below the costal margin, pre-hepatic diameter $(P F D=18 \mathrm{~cm})$, rough phenomena of portal - hepatic decompensation, low pulsatile pedis arteries.

From a biological point of view there are found the following: cholestasis, hepatoprive syndrome, prolongation of the prothrombin time (the patient being currently at home under oral anticoagulant treatment), azote retention syndrome, discreet muscular lysis syndrome, hyperglycaemia, bicytopenia (normocytic hypochromic anaemia + thrombocytopenia) (Table 1).

The thoracic X-ray shows heart with highly increased dimensions, on view of the diaphragm, radio opaque catheter stimulator placed in the RV; atheromatous aorta with calcareous contour; normal pulmonary transparency (Figure 2).

The electrocardiogram at rest during hospitalization reveals pacemaker rhythm, $\mathrm{AV}=69 / \mathrm{min}$, fusion beats (Figure 3 ).

The abdominal ultrasound reveals a finely granular liver, increased reflectivity, IVC $=29$ $\mathrm{mm}$, elevated hepato-jugular reflux, dilated $\mathrm{ESR}=13 \mathrm{~mm}, \mathrm{VP}=14 \mathrm{~mm}$, Doppler curve on the porta vein with ample variations because of the hepato-jugular reflux, perisplenic ascites liquid, ascites liquid in high quantity between the intestinal ansae; cholestasis with swollen gallbladder and walls thickening; small dimensions kidneys (right kidney $=98 \mathrm{~mm}$, parenchymatous index $=10 \mathrm{~mm}$, left kidney $=$ $100 \mathrm{~mm}$, parenchymatous index $=10 \mathrm{~mm}$ ). Otherwise, the echography shows no other modifications.

The ultrasound performed on the soft parts of the right shin and thigh did not signal any particular evolution aspects, and, as a result of the surgical consult, conservative treatment of the hematoma has been chosen, the surgical excision being unnecessary.

The echocardiography detected pericardial fluid located anteriorly at $9.5 \mathrm{~mm}$, located posteriorly at $24 \mathrm{~mm}$, at the right atrium level $23 \mathrm{~mm}$, with direct diastolic collapse of the right atrium, paradoxical motion of interventricular septum, phase IV central mitral regurgitation, mild aortic regurgitation, phase III tricuspid regurgitation, significant disorders in regional kinetics, moderate right cavity dilation and significant dilation of the left atrium, $\mathrm{EF}=42 \%$ (Table 2). At the same time, 
the presence of the pacing catheter is objectified at the right ventricle apical level.

The anticoagulant oral treatment was ceased, an antibiotic, antalgic and hemostatic treatment was established, associated with the hepatoprotective, antihypertensive, diuretic, phlebotropic treatment, with a quasi-complete reabsorption of the right popliteal hematoma and resuming of walking autonomy. Also, taking into account the presence of the haemorrhagipar syndrome, the basic treatment and the benefit/risk balance have been reassessed, an antiaggregant platelet monotherapy being recommended at home.

The prognosis is reserved, taking into account the possibility of decompensation of all related comorbidities, the appearance of hemodynamic instability, the aggravation of vascular and parenchymatous decompensation of the liver cirrhosis, with the development of encephalopathy phenomena and evolution towards exitus. Currently, the patient is within the Child-Pugh class $B$ (score 7), with a MELD score = 19 and in stage III according to the BAVENO IV consensus [2].

\section{Characteristics of the clinical case}

Past smoking patient (30 PA), past chronic ethanol consumer, hypertensive, with valvular heart and coronary disease, with history of pulmonary thromboembolism and hemorrhagiparous phenomena, with permanent electrical cardiac stimulation (for total atrioventricular block with syncope) and chronic venous pathology, evolving on an advanced chronic hepatopathy and a chronic diabetic and uremic status, which develops hemorrhagic complications as a result of the oral anticoagulant treatment.

Table 1. Laboratory tests performed at the time of hospitalization

\begin{tabular}{|c|c|c|}
\hline Test & Result & Normal values \\
\hline \multicolumn{3}{|c|}{ Complete blood count } \\
\hline Hemoglobin(g/dl) & 9.2 & $13.0-17.3$ \\
\hline Hematocrit (\%) & 30.2 & $39.0-51.0$ \\
\hline Medium erythrocyte volume (fL) & 78 & $79.0-96.0$ \\
\hline $\begin{array}{l}\text { Mean erythrocyte hemoglobin } \\
\text { concentration }(\mathrm{g} / \mathrm{dl})\end{array}$ & 30.5 & $32.0-36.0$ \\
\hline Thrombocytes $\left({ }^{*} 10^{3} / \mu \mathrm{l}\right)$ & 138 & $150-400$ \\
\hline \multicolumn{3}{|c|}{ Biochemistry } \\
\hline Glycemia (mg/dl) & 116 & $80-110$ \\
\hline Urea (mg/dl) & 114 & $18-55$ \\
\hline Creatinine (mg/dl) & 2.0 & $0.72-1.25$ \\
\hline Total bilirubin (mg/dl) & 1.56 & $0.20-1.20$ \\
\hline Direct bilirubin (mg/dl) & 0.93 & $0.0-0.5$ \\
\hline Alkaline phosphatase (U/I) & 160 & $40-150$ \\
\hline LDH (U/I) & 245 & $125-220$ \\
\hline Total cholesterol (mg/dl) & 120 & $130-200$ \\
\hline HDL-C (mg/dl) & 20 & $40-75$ \\
\hline Albumin (g/dl) & 3.1 & $3.5-5.2$ \\
\hline \multicolumn{3}{|c|}{ Coagulation } \\
\hline Prothrombin time (sec) & 16.8 & $10-14$ \\
\hline Prothrombin activity (\%) & 58 & $80-125 \%$ \\
\hline INR & 1.51 & $0.8-1.25$ \\
\hline APTT (sec) & 30.6 & $24-36$ \\
\hline
\end{tabular}

Table 2. Echocardiographic changes 


\begin{tabular}{lc}
\hline \multicolumn{1}{c}{ Characteristics } & Patient values \\
\hline Cardiac frequency (beats/minute) & 69 \\
Left ventricle ejection fraction (\%) & 42 \\
Left ventricular end-systolic diameter $(\mathbf{m m})$ & 55.9 \\
Left ventricular end-diastolic diameter $(\mathbf{m m})$ & 71.5 \\
IVS & 13 \\
Thickness of the left ventricular posterior wall & 13.5 \\
(mm) & \\
\hline
\end{tabular}

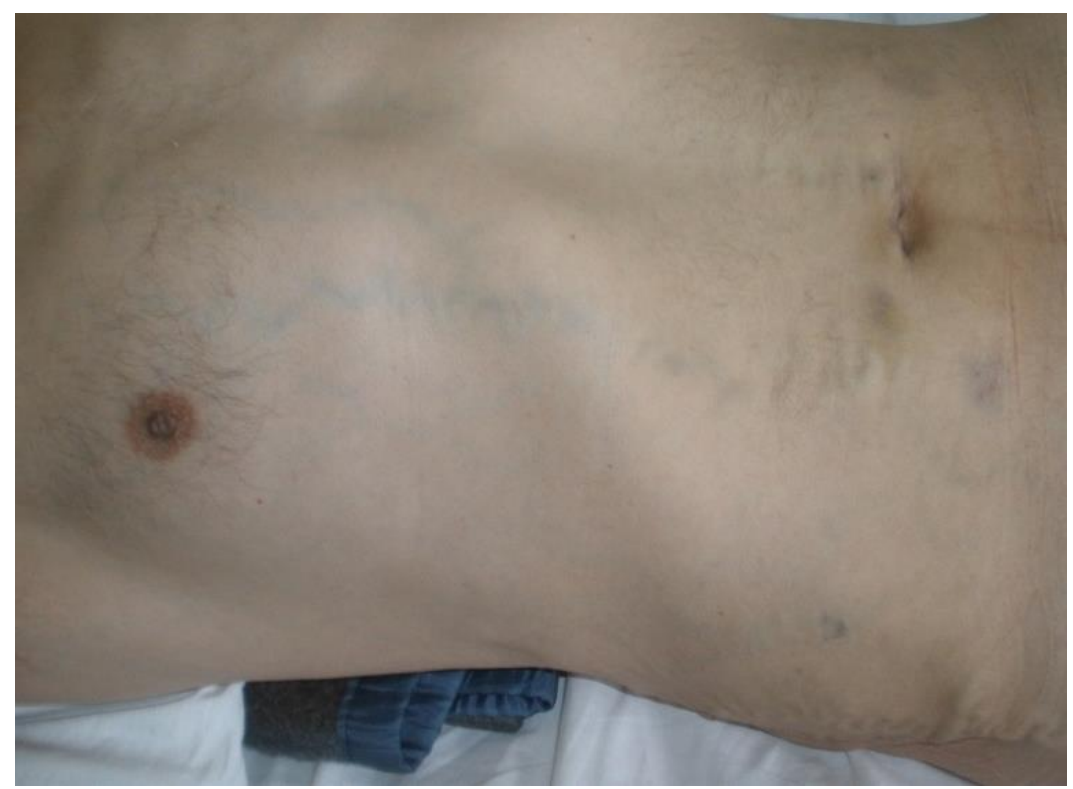

Fig. 1. Objective examination - Thoracic-abdominal collateral circulation

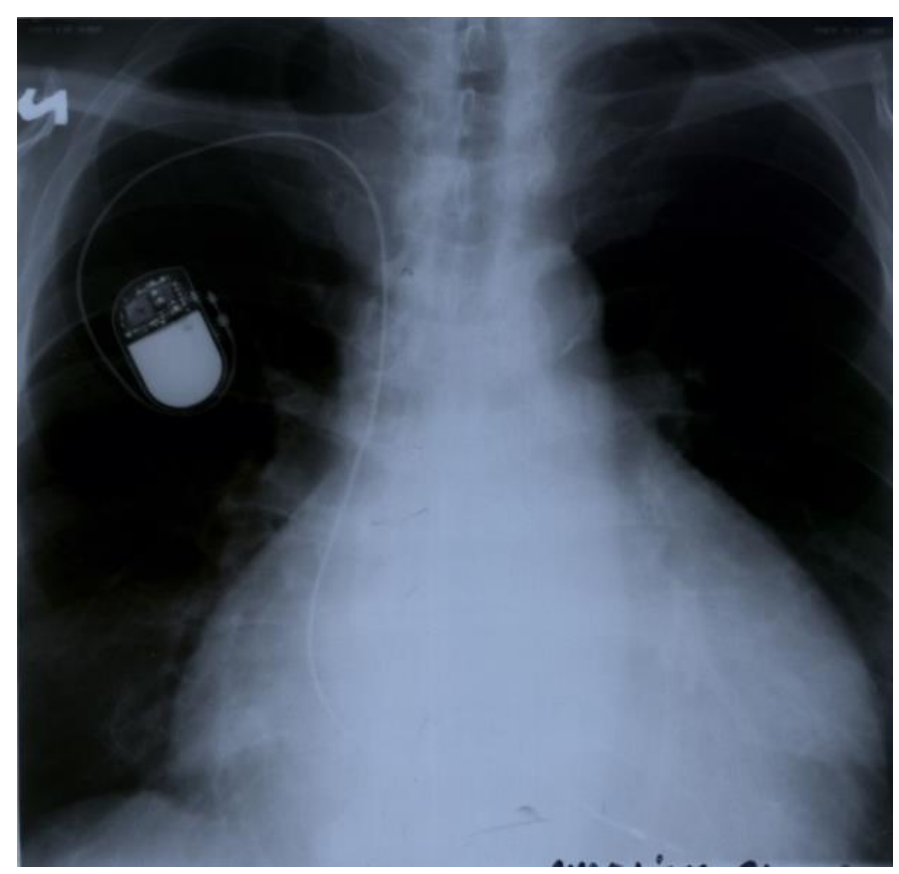

Fig. 2. Thoracic X-ray 


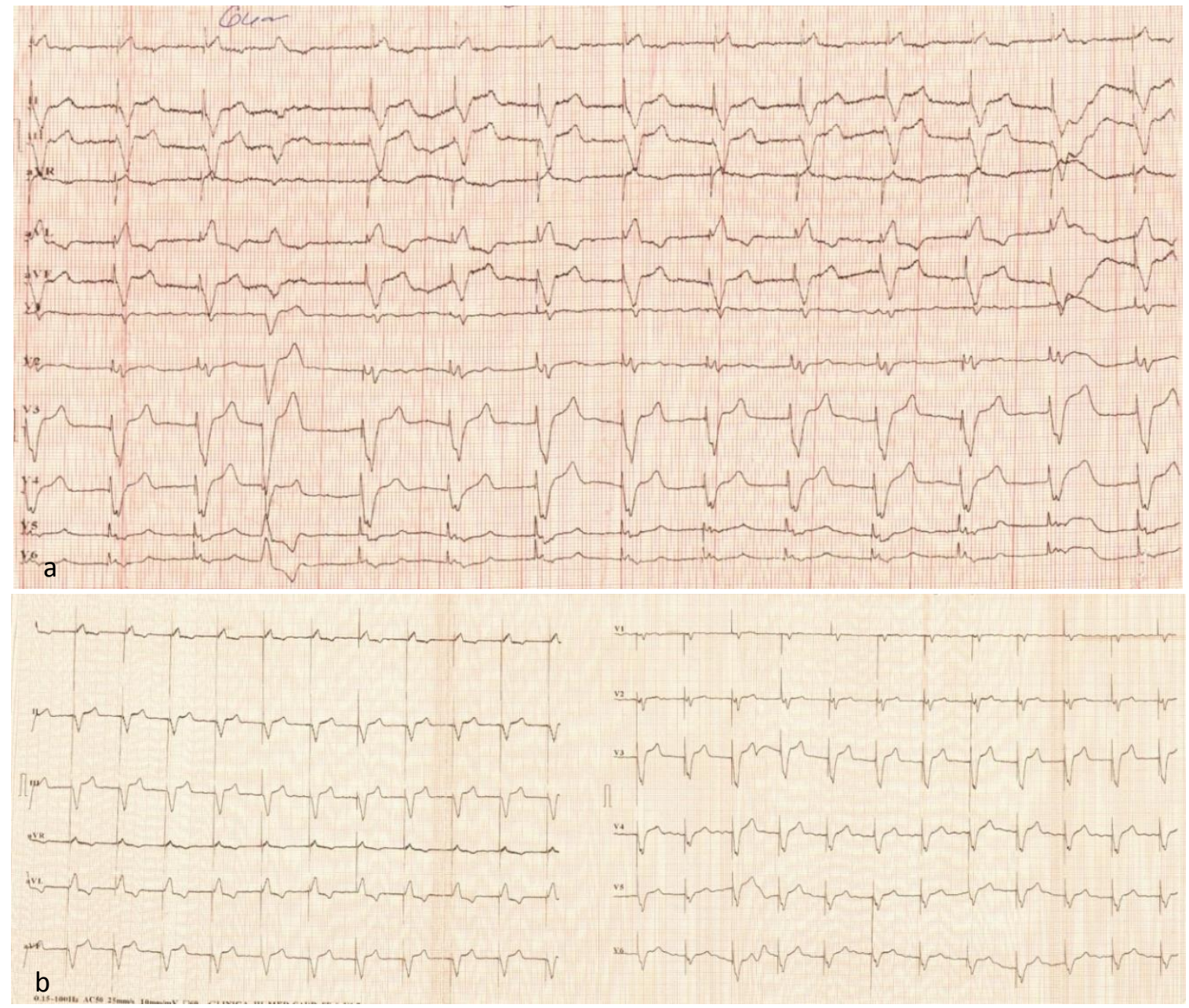

Fig. 3. Electrocardiogram at the time of admission (a) and discharge (b)

\section{Discussions}

Liver cirrhosis is a frequent pathology in daily practice, associating with multiple comorbidities. The liver's role in the hemostasis process is unquestionable, the hepatocyte being the site of synthesis for most of coagulation factors [3]. The liver cirrhosis is associated with an imbalance of the procoagulant and anticoagulant factors, with the possibility of developing venous and arterial thrombosis, having a negative influence on the prognosis [4]. The hemorrhagiparous syndrome presents an increased prevalence in patients with cirrhosis, manifested through: epistaxis, gingivorrhagia, ecchymosis, suffusions, hemorrhaging from the esophagus varices, which are caused by hemostasis changes in patients with severe hepatic pathology. The anticoagulant treatment can be used in curative and/or prophylactic management but establishing some thrombotic and hemorrhagic risk scores and evaluating the benefit/risk ratio are essential. Approximately $30 \%$ of the patients with cirrhosis also develop diabetes mellitus type 2 [5]. Diabetes appears as a complication of cirrhosis, being known as "hepatogenous diabetes", although the term is not acknowledged by the American Diabetes Association and the World Health Organization as a distinct entity [6]. Both the insulin resistance in the adipose and muscular tissue and the hyperinsulinemia seem to be the physiopathological basis of diabetes in chronic hepatopathy [7]. Diabetes mellitus type 2 increases the mortality rate in cirrhotic patients [8]. The treatment for diabetes mellitus type 2 is complex considering the hepatic lesions and the hepatotoxicity caused by oral anti-diabetic medication (most oral anti-diabetic medication is metabolized at hepatic level $[9,10]$. At the same time, hepatogenous diabetes can be considered as an indicator of the worsening of the hepatic dysfunction [11]. The correct assessment of the renal functions and the 
precocious detection of the renal dysfunction in patients with cirrhosis are extremely important for the therapeutic decisions and for prognostic assessments. The presence of renal failure within this group of patients significantly affects the rate of mortality.

\section{Conclusions}

Patients with advanced hepatic affections are highly susceptible to develop hemorrhagic phenomena. Anticoagulation in hepatic affections is currently recommended in portal vein thrombosis, Budd-Chiari syndrome, splenic portal and mesenteric vein thrombosis, deep vein thrombosis and pulmonary embolism [12]. Some authors consider that mild to moderate thrombocytopenia

\section{References}

1. Zatonski WA, Sulkowska U, Manczuk M, et al. Liver cirrhosis mortality in Europe, with special attention to Central and Eastern Europe. Eur Addict Res 2010; 16(4):193-201.

2. Kamath PS, Kim WR, Advanced Liver Disease Study Group. The model for end-stage liver disease (MELD). Hepatology 2007; 45(3):797805.

3. Al Ghumlas AK, Abdel Gader AG, Al Faleh FZ. Haemostatic abnormalities in liver disease: could some haemostatic tests be useful as liver function tests? Blood Coagul Fibrinolysis. 2005; 16(5):329-35.

4. Tripodi A, Mannucci PM. The coagulopathy of chronic liver disease. N Engl J Med 2011; 365(2):147-156.

5. Hickman IJ, Macdonald GA. Impact of diabetes on the severity of liver disease. Am J Med 2007; 120(10):829-834.

6. Holstein A, Hinze S, Thiessen E, Plaschke A, Egberts $\mathrm{EH}$. Clinical implications of hepatogenous diabetes in liver cirrhosis. $J$ Gastroenterol Hepatol 2002; 17(6):677-681.

7. Steppan CM, Bailey ST, Bhat S, et al. The hormone resistin links obesity to diabetes. Nature 2001; 409(6818):307-312.

8. Nishida T, Tsuji S, Tsujii M, et al. Oral glucose tolerance test predicts prognosis of patients (>50,000/mmc) does not represent a contraindication in venous thromboembolism prevention, in specific conditions, as neoplasia [13]. The multivalent clinical approach of the patient with cirrhosis, in relation with therapeutic and prognostic implications, underlines the necessity to actively monitor this group of patients [14]. Taking a therapeutic decision should include correctly assessment of all clinical evidence of both clinical indications and contraindications. Furthermore, the research perspectives should studying the existence of prognostic correlations between coagulogram values and MELD and Child-Pugh scores, the role of diabetes mellitus type 2 in chronic hepatopathies genesis, the impact of hepatogenous diabetes in the natural history of patients with liver cirrhosis.

with liver cirrhosis. Am J Gastroenterol 2006; 101(1):70-75.

9. Tolman KG, Fonseca V, Dalpiaz A, Tan MH. Spectrum of liver disease in type 2 diabetes and management of patients with diabetes and liver disease. Diabetes Care 2007; 30(3):734743.

10. El-Serag HB, Everhart JE. Diabetes increases the risk of acute hepatic failure. Gastroenterology 2002; 122(7):1822-1828.

11. Del Vecchio Blanco C, Gentile S, Marmo R, Carbone L, Coltorti M. Alterations of glucose metabolism in chronic liver disease. Diabetes Res Clin Pract 1990; 8(1):29-36.

12. Roberts LN, Patel RK, Arya R. Haemostasis and thrombosis in liver disease. Br J Haematol 2010; 148(4):507-521.

13. Tufano A, Guida A, Di Minno MN, Prisco D, Cerbone AM, Di Minno G.. Prevention of venous thromboembolism in medical patients with thrombocytopenia or with platelet dysfunction: a review of the literature. Semin Thromb Hemost 2011; 37(3):267-74.

14. Moller S, Henriksen JH. Cirrhotic cadiomyopathy. J Hepatol 2010; 53(1):179190. 